\title{
Violences sociales et exclusions réciproques en milieu urbain africain. Le cycle des échanges de violence dans les rapports familiaux
}

Sylvie Ayimpam

\section{(2) OpenEdition}

Journals

\section{Édition électronique}

URL : http://journals.openedition.org/apad/2993

ISSN : 1950-6929

\section{Éditeur}

LIT Verlag

Édition imprimée

Date de publication : 4 avril 2008

Référence électronique

Sylvie Ayimpam, «Violences sociales et exclusions réciproques en milieu urbain africain. Le cycle des échanges de violence dans les rapports familiaux », Bulletin de l'APAD [En ligne], 27-28 | 2008, mis en ligne le 18 juin 2008, consulté le 07 septembre 2020. URL : http://journals.openedition.org/apad/2993

Ce document a été généré automatiquement le 7 septembre 2020.

Bulletin de l'APAD 


\title{
Violences sociales et exclusions réciproques en milieu urbain africain. Le cycle des échanges de violence dans les rapports familiaux
}

\author{
Sylvie Ayimpam
}

1 A l'encontre des discours sur la violence centrés sur une dichotomie exclusive victimes/agresseurs, j'essaie dans cette contribution de mettre en lumière le caractère réciproque et cyclique des violences ordinaires, dans le contexte familial en milieu urbain « kinois ». A partir de deux études de cas réalisées sur base d'histoire de vie à Kinshasa, j'ai choisi d'investiguer un type particulier de lien familial, le rapport épouse/belle famille ainsi que ses violences subséquentes. Ayant par principe, la figure d' « étrangère » parce que provenant d'une autre famille, " acquise » au moyen d'une dot et placée par conséquent dans une relation d'infériorité vis-à-vis de son mari et de la parentèle de celui-ci, l'épouse entretient souvent, un rapport dialectique avec ces derniers. Et pourtant une telle description, prompte à donner une figure de « victimes " aux épouses, cache une réalité sociale plus complexe, où chacun à son tour peut être victime et oppresseur, et surtout développer des stratégies de résistance et d'exclusion. Sans nier que les violences subies par les épouses de la part de leurs belles familles, peuvent s'inscrire dans le panel des "violences contre les femmes " ${ }^{1}$, il convient sans doute également d'analyser cette violence dans une perspective diachronique. A travers la relation épouse/belle-famille, c'est d'abord et surtout le lien familial et ses violences associées que je voudrais interroger.

2 L'univers familial en milieu urbain africain, comme démontré remarquablement par Pierre Janin et Alain Marie (2003), peut être un microcosme de la violence. Si je m'inscris dans la continuité de leurs analyses à propos de la réciprocité de la violence familiale, j'essaie en revanche d'approfondir l'aspect de la reproduction de la violence et de la perpétuation des rapports de violence dans le contexte familial. La famille est un espace social qui se prête particulièrement à l'observation et à l'étude des violences 
qui prennent place dans le quotidien des rapports sociaux ordinaires. La famille africaine, parce qu'elle s'inscrit presque toujours dans une logique communautaire des rapports sociaux, parce qu'elle se pense toujours grande et composée de relations hiérarchisées et complexes entre consanguinité et affinité, entre grande proximité et petite proximité, peut être un laboratoire de violence, prédisposant les membres de la famille à subir autant qu'à concourir à cette violence.

Ceci étant, mon propos est de montrer que la violence dans les rapports familiaux se reproduit, puisque ces rapports sont basés sur une structure fortement hiérarchisée, érigeant les rapports familiaux en rapports d'autorité. Mais puisque les positions dominantes de la structure familiale sont réversibles et relatives à certaines situations, alors cette violence ne peut qu'être réciproque et cyclique. Un acte de violence observé, un événement que chacun commente, n'est souvent qu'un moment particulier d'un cycle complexe. La reconstitution des moments importants du cycle requiert une approche diachronique de l'observation situationnelle. Cet article s'appuie ainsi sur deux récits de vie, sélectionnés pour leur exemplarité et parce qu'ils réunissent suffisamment d'informations permettant, sinon de reconstituer un cycle complet d'échanges de violences, au moins d'en décrire différentes séquences remarquables. ${ }^{2}$ Le temps, la proximité et la confiance ont été nécessaires pour réunir les informations constituant ces récits, qui sont faits du témoignage des différentes personnes ayant accepté de «livrer » leurs expériences vécues, leurs perceptions et par-là même de dévoiler certains secrets de famille. ${ }^{3}$

Violences réciproques, violences cycliques : À la violence de l'autorité, la violence de la résistance

En mars 2001 ont eu lieu à Kinshasa, les funérailles de Papa Mapasa ${ }^{4}$, un ancien fonctionnaire à la retraite. La veillée mortuaire avait lieu dans la cour familiale située dans un quartier populaire à l'est de la ville de Kinshasa. Cette veillée durait depuis près de seize heures, et tirait presque à sa fin, puisqu'on approchait de l'heure de l'inhumation. Mama Mapasa, sa veuve, était assise sur une natte placée à proximité du cercueil, la tête recouverte d'une pièce de drap blanc. Elle n'avait pas mangé depuis vingt quatre heures. Elle semblait très faible et fatiguée, pourtant, ses belles sœurs assises face à elle, l'apostrophaient, lui reprochant de ne pas avoir assez pleuré :

«Pleure, mais pleure donc! C'est toi qui bouffais son argent, non! Es-tu fatiguée de pleurer? Tu ne te lèveras que lorsque tu auras rempli ce seau (placé près d'elle) de tes larmes!".

5 Malgré un besoin pressant d'aller aux toilettes, la «coutume» lui interdisait, semble t-il, de se lever. Il faudrait qu'elle pleure suffisamment pour que sa belle famille lui accorde la permission de se lever, d'aller aux toilettes, de boire ou de manger. Toute la nuit passée, chaque fois qu'elle était emportée par le sommeil, on la secouait en lui disant de pleurer son mari avant de dormir. Face aux murmures de protestation qui montaient de l'assemblée, une de ses belles sœurs, veuve elle aussi, justifia cette attitude ainsi :

«C'est la tradition, c'est comme ça, on n'y peut rien. Elle n'est ni la première, ni la dernière. Moi aussi, quand j'ai perdu mon mari, on m'a fait la même chose. Mais moi, j'ai bénéficié d'un traitement de faveur, parce que je m'étais montrée bonne et généreuse envers ma belle famille du vivant de mon mari. En retour, ils n'ont pas été trop durs avec moi. Mais votre fille, elle, a toujours été arrogante et chiche! Elle ne se lèvera qu'après avoir rempli ce seau (de ses larmes) ! .

6 La famille de Mama Mapasa s'échauffa à son tour, indiquant que les belles-sœurs avaient dépassé les limites. Mais la belle famille resta intransigeante. Mama Mapasa fait 
partie d'une église dont le pasteur et les adeptes étaient venus lui apporter soutien et réconfort depuis les débuts des funérailles. Une femme se détacha du groupe des fidèles de l'église et lança à la belle-famille :

«La tradition, la tradition, c'est quoi? Aujourd'hui, nous sommes tous chrétiens. Notre tradition aujourd'hui, c'est le christianisme. Vous devriez avoir honte de pratiquer ces choses de la tradition qui ne sont pas compatibles avec la dignité humaine!»

7 Une des belles-sœurs de Mama Mapasa, qui avait apparemment le même âge que cette femme, lui répondit :

« Eh bien! Si tu penses que la tradition n'a plus d'autorité, toi, viens la prendre de force et emmènes-la aux toilettes. Et on verra si c'est toi qui vas la «laver» après l'enterrement!».

8 En effet, en cas de décès du conjoint, la famille de ce dernier détient une arme puissante et redoutable contre sa veuve : le rite de purification. Ce rite se fait, entre autres, à travers une toilette purificatrice qui aurait pour but, selon les conceptions locales, de séparer définitivement les deux conjoints qui ont été unis par les liens du mariage. ${ }^{5}$ Aussi, devant un défi si inattendu et une menace à peine voilée de ne pas « laver» (purifier) la veuve, la dame se rétracta. Optant pour la négociation, le pasteur et un des aînés de la famille de Mama Mapasa, entamèrent une longue discussion avec la belle-famille. Finalement, les belles-sœurs de Mama Mapasa acceptèrent que leur soit versée une certaine somme d'argent contre l'autorisation accordée à la veuve d'aller aux toilettes, de manger et de boire. ${ }^{6}$

9 La maltraitance dont cette veuve a fait l'objet durant les funérailles de son mari, est une histoire banale parmi tant d'autres en ville. ${ }^{7}$ À Kinshasa, les funérailles du conjoint sont généralement un moment redouté par les femmes mariées, car cela peut être l'occasion pour la belle-famille de se venger, d'infliger une "correction »à une épouse qui ne se serait pas soumise à elle du vivant de son mari. La hantise pour une veuve de ne pas être " purifiée » par la belle-famille et donc de continuer à être liée au conjoint, la tient soumise et assujettie aux maltraitances de la belle-famille pendant toute la période des funérailles. La maltraitance des veuves (ou des veufs) durant les funérailles du conjoint se présente comme une pratique sociale admise, tolérée, ou tout au moins « comprise » par les uns et les autres. L'expression " c'est la tradition » utilisée comme justification, indique que l'on est en présence des pratiques normées, réglées. Au nom de la «tradition», des traitements dégradants et humiliants sont parfois infligés aux veuves ou aux veufs tout au long des funérailles, traitements qui dans beaucoup de cas n'ont plus rien à voir, ni dans l'esprit ni dans la pratique, avec la «tradition» dont ils se réclament. ${ }^{8}$ Très peu de gens s'arrêtent pour réellement interroger, mettre en question de telles pratiques, sinon lorsqu'elles dépassent « certaines limites acceptables ». Mais à partir de quelles limites peuvent-elles être acceptées ou refusées? Que fait-on lorsque les limites acceptables sont dépassées? Dans le cas de Mama Mapasa, il n'y a pas eu d'opposition ni de révoltes, mais plutôt des négociations suivies d'un marchandage pour les ramener dans les limites de «l'acceptable». Mais ce que je viens de relater n'est qu'un moment du cycle d'échange de la violence, un acte de vengeance, un événement qui ne peut être compris s'il n'est pas mis en rapport avec l'ensemble des événements antérieurs qui tissent l'histoire des relations problématiques entre Mama Mapasa et sa belle famille. Quand on accède à cette histoire, la réciprocité des échanges violents apparaît clairement, obligeant le chercheur à dépasser le point de vue synchronique, qui érigerait les uns en agresseurs exclusifs et les autres en victimes 
exclusives. En fait, le cycle des échanges violents est enclenché de longue date et il se poursuivra comme on verra, bien après les funérailles.

La source des mésententes entre Mama Mapasa et sa belle-famille, c'est avant tout la désapprobation de son choix en tant qu'épouse de leur « fils ». En effet, la mère de Papa Mapasa espérait secrètement que ce dernier épouserait la fille de sa meilleure amie. Le père de Papa Mapasa ayant donné son accord malgré l'avis contraire de sa femme, le mariage eut lieu. C'était le début d'une longue histoire ambiguë. A l'époque de leur mariage en 1960, Papa Mapasa était encore étudiant à l'université. Faute de ressources, le couple dut se résoudre à habiter dans un "deux pièces " que la famille de Papa Mapasa lui avait alloué dans la grande cour familiale ${ }^{9}$. Mama Mapasa qui travaillait déjà à cette époque comme infirmière à l'hôpital général de Kinshasa, essaya en vain de convaincre son mari de quitter cette cour familiale où les rapports quotidiens étaient très difficiles pour elle.

11 En effet, les rapports entre Mama Mapasa et sa belle-mère sont restés très tendus tout au long des sept années pendant lesquelles ils résidèrent dans la cour familiale. La belle-mère ne manquait aucune occasion de lui montrer son hostilité, la traitant avec mépris et condescendance. Tout était occasion pour se moquer de Mama Mapasa ou pour la ridiculiser. ${ }^{10}$ Un jour, fatiguée d'être traitée de la sorte, elle "osa » dire à sa belle-mère que son comportement était injuste et discriminatoire à son égard. La belle-mère considéra ce reproche comme un affront. Elle lui rétorqua qu'elle n'avait pas le droit de lui parler de la sorte, puisqu'en tant que sa belle-fille, elle lui devait soumission et respect inconditionnels. "Estime-toi heureuse que je ne me sois pas montrée aussi dure et méchante envers toi que l'avait été ma belle-mère à mon égard!», lui dit-elle. Considérant que sa belle-fille n'avait pas le droit de lui faire de reproche, elle rapporta à tous ses enfants que cette dernière l'avait «insultée». Alors, les uns après les autres, ils se dressèrent contre Mama Mapasa. Les rapports quotidiens devinrent très difficiles : menaces, disputes, insultes, conflits, etc.

Les attitudes de la belle-mère et de la belle-fille durant cette séquence, sont particulièrement éclairants. Ils illustrent l'impératif hiérarchique des rapports sociaux qui ordonne une société où règne la logique communautaire. Ces violences quotidiennes dans les rapports familiaux sont souvent l'expression des rapports sociaux fondés sur la domination. Elles ne sont donc pas spécifiques aux rapports entre belle-mère et belle-fille. Dans les organisations familiales fortement hiérarchisées, elles affectent de même les autres relations dominant/dominé telles les relations aînés/ cadets. Cette violence qui imprègne les rapports familiaux au quotidien peut ici être définie comme "toute action verbale, physique ou psychologique, imparfaitement régulée, qui dégrade un rapport hiérarchique ou une relation plus égalitaire vers une plus grande domination et soumission »(Janin $2003: 33)$. Tout acte qui dans les rapports sociaux quotidiens vise à infliger à autrui de manière intentionnelle une souffrance psychique (la peur, l'humiliation, l'anxiété, le désespoir) ou une souffrance physique (des sévices ou des lésions corporels) sera considérée comme acte de violence.

13 Les relations entre les membres de la hiérarchie familiale, entre les générations successives, sont ordonnées par un autoritarisme et un pouvoir dont les attributs tiennent à la légitimité d'exercice d'une violence coercitive, précisément parce que l'adhésion de ceux qui sont au bas de la hiérarchie non seulement ne va pas de soi, mais elle n'est même pas explicitement recherchée. Les positions d'autorité se contentent généralement de recourir à la force brute pour faire exécuter leur volonté, mais aussi, 
et surtout, les plus puissantes d'entre elles disposent de manière quasiment exclusive $\mathrm{du}$ recours aux forces occultes (Janin et Marie 2003). Cette coercition permanente qu'exerce la menace de recourir à des violences occultes contribue de manière souvent invisible (à l'extérieur de la famille) au maintien des rapports familiaux de domination. Mais c'est d'abord la socialisation, à laquelle les femmes travaillent de manière active, qui conditionne les membres de la hiérarchie familiale à se soumettre à ce type de rapport. ${ }^{11}$ Elle permet l'intériorisation par les membres de la famille des valeurs culturelles légitimant les rapports de domination. Ceux qui sont au bas de la hiérarchie sont censés devoir une soumission inconditionnelle à ceux qui sont en haut de la hiérarchie. Ceux qui sont en position d'autorité essaient, bien sûr, de perpétuer et légitimer leur domination en invoquant notamment la tradition.

Violences réciproques, violences cycliques : Victimes hier, agresseurs aujourd'hui

14 En 1968, dès que son mari décrocha son diplôme universitaire et trouva un bon emploi, Mama Mapasa le pressa de quitter la cour familiale. Le couple s'installa donc dans une maison en location-vente, qui devint leur propriété quinze ans plus tard. Mama Mapasa qui avait déjà six enfants, lorsqu'ils s'installèrent dans leur maison, refusa d'accueillir, d'autres « enfants » de la famille de son mari qu'on essayait de leur confier :

«Ils m'avaient mis mal à l'aise chez eux, alors je n'allais pas les laisser venir me mettre mal à l'aise chez moi ! Ils voulaient que je sois gentille et généreuse avec eux, quand ils venaient nous voir, oubliant tout ce qu'ils m'avaient fait subir! Ils voulaient que je leur donne à manger, à boire, que je leur sourie, alors que quand j'étais dans la grande cour familiale, ils m'avaient tous malmenée »

Néanmoins, un beau jour, sans solliciter leur avis, sa belle-mère amena deux nièces de son mari âgées de dix et douze ans, filles d'une des sœurs aînés de Papa Mapasa. Mama Mapasa interpréta cet acte comme un moyen par lequel sa belle-famille essayait de garder une main mise sur elle et sur son foyer. Elle se vengea donc sur les deux enfants à qui elle rendit la vie difficile. La mère des deux filles, me confiait ceci : "Mes filles étaient des esclaves, des bonnes à tout faire, dans la maison de leur propre oncl ». Selon elle, Mama Mapasa aurait torturé ses enfants physiquement et moralement: elles auraient vécu dans la terreur. Elle les criblait des corvées domestiques et autres tâches ménagères. Tout était prétexte pour les battre, les insulter, les menacer régulièrement, etc. Elles étaient mal vêtues, mal nourries. Elles n'avaient pas le temps de faire leurs devoirs scolaires et de réviser leurs leçons, à cause des corvées domestiques et cela se ressentait sur leurs résultats scolaires. Mama Mapasa ne ratait pas l'occasion alors d'en rire méchamment et de les traiter d'idiotes. Et les deux filles se gardaient bien de révéler à la famille tout ce qu'elles subissaient dans cette maison par peur des représailles de Mama Mapasa, qui menaçait par ailleurs, de les tuer si elles osaient parler.

Quant au silence et à l'indifférence de Papa Mapasa devant l'attitude de sa femme envers ses deux nièces, ils sont interprétés par sa sœur comme l'effet de la domination que sa femme aurait eu sur lui après qu'ils aient quitté la grande cour familiale, grâce à l'usage de certains fétiches qui auraient assujetti le mari à sa seule volonté. Pour elle, il n'était que justice que Mama Mapasa paie pendant les funérailles de son mari tout ce qu'elle avait fait subir à sa belle-famille pendant la période où elle était en position de pouvoir.

"Cette femme ne nous respectait plus et ne nous traitait pas selon le respect dû à une belle-famille, quand nous allions chez elle. Elle était arrogante, insoumise et nous traitait de haut. Elle semblait oublier qu'un jour, notre tour allait arriver ». 
Ainsi justifiait-elle et légitimait-elle la maltraitance dont Mama Mapasa avait fait l'objet pendant les funérailles de son mari. Pour Mama Mapasa, l'attitude hostile envers les deux filles et envers sa belle-famille qu'on lui reprochait, ne relevait que d'une stratégie de résistance face à une oppression subie antérieurement qu'elle ne voulait plus voir se répéter. Mais la vengeance de la belle-famille ne s'est pas arrêtée aux funérailles. Après le deuil, Mama Mapasa et ses enfants ont été dépouillés de tous les biens laissés par son défunt mari: maison, voitures, meubles, appareils électroménagers, voire cadeaux personnels tels que bijoux, vêtement ou vaisselle, etc. La famille de Papa Mapasa n'eût pas de compassion non plus pour les orphelins, les enfants de leur "frère ", leur propre «sang». Ils furent tous mis à la porte, en même temps que leur maman, malgré l'intervention de certains notables du quartier et des pasteurs. Mama Mapasa dût se réfugier temporairement chez ses parents, en attendant de trouver une petite maison à louer.

Il est intéressant de noter que ni la veuve, ni ses enfants, n'avaient vraiment insisté pour réclamer l'héritage lorsque la belle-famille les a mis à la porte et confisqué tout l'héritage. Aucune action juridique n'a été entreprise par la veuve et les orphelins pour réclamer leur droit, alors que le code de la famille au Congo règle assez clairement la question de la succession : les orphelins sont les héritiers de premier rang ayant droit aux trois quarts de l'héritage, et la veuve et les membres de famille du défunt sont les héritiers de second rang, devant se partager le quart restant de l'héritage. Il est de plus en plus courant à Kinshasa d'observer la veuve (quelquefois le veuf) et les orphelins être complètement dépouillés de l'héritage, chassés de leur maison. Très peu sont ceux qui tentent de réclamer leur droit ou qui essaient de tenir tête face à la famille du père ou de la mère décédés, confisquant l'héritage. L'on observe plutôt une attitude de résignation, d'apathie, comme si cela était une fatalité. Interrogée à ce sujet Mama Mapasa a dit ceci :

"Ce sont des biens que nous avons trouvés ici sur terre, et je ne voudrais pas me battre à cause de cela. Mon mari qui les avait acquis, est mort sans pouvoir les emporter. Ceux qui les confisquent aujourd'hui, mourront aussi sans pouvoir les emporter. J'ai donné instruction à mes enfants de ne pas se battre à cause de cela. Je ne sais pas ce que la famille de mon mari a dans le ventre. Alors, je ne voudrais pas que moi ou mes enfants, perdions nos âmes à cause de cela. La vie de mes enfants a beaucoup plus de prix à mes yeux que tous les biens que l'on nous a pris ».

Il ressort des propos de Mama Mapasa qu'elle a eu peur que ses enfants ne soient ensorcelés par leur famille paternelle au cas où ils réclameraient l'héritage ou qu'ils contesteraient leurs décisions. L'expression " je ne sais pas ce qu'ils ont dans le ventre " veut dire en d'autres termes «je ne sais pas s'ils sont sorciers ${ }^{12}$ Ce que l'on peut parfois prendre pour de l'incurie ou de la lâcheté face à une injustice criante, la résignation observée, n'est bien souvent que la peur de la sorcellerie des autres. On peut se demander pourquoi certaines personnes, qui ont en leur faveur des textes légaux (le Code de la famille), qui peuvent avoir à leur disposition la machine judiciaire (les cours et tribunaux), se laissent dépouiller sans réclamer leur droit, sans intenter de procès, acceptent tête baissée des décisions aussi injustes. La peur d'être ensorcelés fait que depuis des générations l'on observe en ville des gens se faire dépouiller totalement de leur droit à l'héritage..$^{13}$ On rapporte parfois pour justifier une telle passivité, les malheurs qui seraient arrivés aux enfants dans telle ou telle autre famille, parce qu'ils avaient osé réclamer leur héritage : les enfants morts les uns après les autres, des maladies incurables, l'incapacité pour les filles à trouver des maris, ou d'avoir des 
enfants, l'incapacité de finir les études pour les garçons, etc. Longue est la liste des malheurs qui risquent d'arriver en cas de contestation, malheurs dont les victimes ont la hantise. Dans certains cas, des veuves et des orphelins téméraires qui tentent de s'opposer à ces décisions injustes, ou qui souhaiteraient les contester, sont découragés dans leurs initiatives par des proches ou des parents, qui brandissent les spectres des malheurs qui peuvent leur arriver s'ils continuaient leur entreprise de contestation. Les rapports de pouvoir qui fondent essentiellement les rapports familiaux dans les univers communautaires, se déclinent au moyen de la violence entendue comme «toute coercition physique ou morale "légitimée » par le droit du plus fort ». (Janin et Marie 2003 : 5) Ici, ceux qui sont en position de force, sont censés détenir des forces occultes (en l'occurrence la sorcellerie) grâce auxquelles, ils peuvent obtenir la soumission ou arracher l'adhésion des plus faibles à des décisions injustes. Il s'agit ni plus ni moins d'un abus de pouvoir.

20 En juillet 2001, quatre mois après le décès de Papa Mapasa, sa fille aînée, Chantal, qui avait été demandée en mariage par un riche négociant malien une année plus tôt, se préparait à célébrer son mariage. Contrairement à ce qui se fait d'ordinaire, Chantal, sa maman, et la famille de la maman, décidèrent d'un commun accord d'exclure la famille de Papa Mapasa des tractations pour le payement de la dot et des cérémonies de mariages, coutumier, civil et religieux. Mama Mapasa en voulait tellement à sa belle-famille de les avoir mis à la rue ses enfants et elle, qu'elle refusa catégoriquement malgré les conseils, de les associer au mariage de sa fille. Le mariage coutumier eût lieu en août 2001. Sans y avoir été conviée, sa belle-famille s'y présenta quand même, mais elle les chassa sans ménagement. Elle leur dit ouvertement qu'ils ne "boufferaient " pas la dot de sa fille, puisqu'ils l'avaient dépouillée de son héritage. Hués par l'assemblée venue à la cérémonie coutumière et par les voisins, ils se sentirent obligés de partir mais non sans proférer des menaces à l'encontre de Mama Mapasa : «Si c'est vraiment de la fille de notre frère que tu nous refuses la dot, on verra !». Après trois ans de mariage, Chantal et son mari n'arrivaient pas à concevoir d'enfant. Elle se mit à soupçonner que cela put être dû au mécontentement de la famille de son père qui n'avait pas reçu son dû : la dot. En novembre 2004, Chantal se résolut à aller demander pardon à ses oncles et tantes paternels, et son mari dût leur payer une nouvelle dot. Deux mois plus tard, Chantal tomba enceinte. Sa mère, Mama Mapasa me dit : « Ne te disais-je pas que ces gens doivent avoir quelque chose dans le ventre? C'est cela qui leur permet de faire du mal aux autres sans se repentir, de moissonner là où ils n'ont pas semé. Mais cela ne fait rien. Un jour, ils finiront par payer tout ce qu'ils font aux autres».

21 Contrairement à Mama Mapasa, Tabou a entretenu quant à elle des rapports plus ou moins pacifiques avec sa belle-famille, mais aussi avec son mari Kola pendant les premières années de son mariage. Les choses ont commencé à se "gâter " entre les époux lorsqu'elle a décroché un emploi dont le salaire était supérieur à celui de son mari. Ensuite, les tensions se sont exacerbées lorsque la sœur aînée de Kola, Kato chassée par sa belle-famille, vint se réfugier chez eux. Le mari de Kato était un ingénieur civil, ayant brillamment terminé ses études, mais n'ayant jamais réussi à trouver un emploi. La belle-famille de Kato, ayant tant investi en ce fils aîné et fondé sur lui ses espoirs, s'est trouvée désemparée face à cette situation. Il y avait certainement une raison et elle entreprit de découvrir qui le «bloquait ». ${ }^{14}$ Une de ses soeurs, très engagée dans une église, finit par «découvrir» en consultant son pasteur, 
que c'était le fils aîné de son frère, un enfant maladif, qui était le fameux sorcier "bloqueur». Ce dernier tiendrait sa sorcellerie de sa maman, Kato, qui serait elle même, une sorcière «inconsciente " (premier acte de violence psychique contre kato). On tint le mari de Kato au courant des faits et on lui demanda de chasser sa femme afin d'être « délivré » de ces «blocages » (deuxième acte de violence psychique sur le mari et sur Kato). N'ayant pas le courage d'en parler à son épouse ni de lui exprimer ouvertement son ressentiment, le mari de Kato devint très violent et se mit à la battre régulièrement, espérant ainsi qu'elle fuirait le toit conjugal d'elle-même (troisième acte de violence physique sur Kato). Mais encore amoureuse de son mari, Kato n'en fit rien. Elle se retrouva parfois avec des foulures, des brûlures, des plaies ouvertes, des yeux tuméfiés, des fractures, etc., sans pouvoir s'expliquer la violence soudaine de son mari ${ }^{15}$. Elle supposait qu'elle était peut-être due à ses déboires face à la vie. Cependant, le mari hésitait toujours à la chasser. D'une part, n'ayant pas de certitude sur la sorcellerie supposée de sa femme, il était partagé entre le doute et le ressentiment. Par ailleurs, Kato le soutenait financièrement depuis ses six années de chômage. Etant sans ressources, sa famille lui proposait de revenir vivre à la maison, une fois la femme partie.

En revanche, chaque fois que Kato était battue par son mari, elle répondait à son tour en le privant de nourriture et d'argent de poche. C'était, d'après elle, le seul moyen de pression efficace pour contrer cette violence conjugale, mais aussi une forme de coercition à opposer à la violence de son mari ${ }^{16}$. Apparemment la "technique " marchait, mais ses effets ne duraient guère. Face à la privation de nourriture et d'argent de poche, le mari se calmait, pendant quelques semaines, puis il recommençait à la battre. Cela dura deux ans. Kato avait donc réussi à développer une stratégie de résistance face à son mari : à la violence de la force physique, elle opposait une autre force, réciproque, une forme de rétorsion silencieuse: le priver de nourriture et d'argent de poche alors qu'il n'avait pas de ressources. Cette séquence pose un problème théorique intéressant. Il s'agit de savoir si une force qui s'oppose à «la violence » peut être qualifiée de "violence ».

Est-ce qu'un acte qui s'oppose à un autre acte essayant d'infliger intentionnellement une souffrance physique ou psychique à une personne, est une violence ? Peut-on dire ici que le mari et la femme endossent en même temps le rôle d'agresseurs et de victimes? Du point de vue de la philosophie morale et politique, si l'on appelle « violence » l'acte de Kato, alors, la question d'une légitimité possible de la violence est posée. Par contre si l'on dénie le terme de violence à son acte, pour réserver ce terme aux actes posés par le mari (qui correspondent à la définition adoptée ici de la violence), alors une force (celle de la femme) qui empêche le déploiement de la violence aveugle du mari est une force d'ordre, c'est-à-dire le contraire même de la violence. D'autre part, l'ordre des actes dans le cycle de réciprocité est-il indifférent? Un acte initial du mari vise à infliger une souffrance physique et psychique à Kato qui réagit (donc réciproquement) en posant un acte qui vise à empêcher son mari de perpétuer cette souffrance. Devant les tergiversations du mari à chasser sa femme, la belle-famille entreprit de le faire elle-même en 1992 à l'issue d'une grande réunion de famille. La belle-famille proclama publiquement que Kato était une sorcière, qu'elle avait transmis cette sorcellerie à son fils aîné et que la mère et le fils «bloquaient » la réussite du mari (quatrième acte de violence psychique et physique contre Kato). Chassée, Kato alla se réfugier chez son frère Kola, avec ses enfants. Arrivée dans la maison de son frère pleine de rancœur, l'épouse de son frère, Tabou, se présenta alors, comme une 
« victime » propice sur qui déverser toute l'humiliation et l'injustice subies. Quant à Tabou, elle accepta difficilement la présence de sa belle-sœur et de son enfant, présumés sorciers, craignant qu'ils ne " contaminent $»^{17}$ ses propres enfants ou qu'ils ne les attaquent en sorcellerie.

Cette attitude de Tabou est l'effet direct du dernier acte de violence psychique et physique posé par la famille du mari de Kato (la proclamation publique de sa nature sorcière). Tabou a peur : la peur est un des effets produits par une violence psychique efficace. L'une et l'autre vont développer des stratégies de violence et d'exclusion réciproques. L'affrontement de violences entre Kato et Tabou est très intéressant parce que symétrique et réciproque : ces deux femmes ont des droits exclusifs et inaliénables mais différents sur le même homme Kola. Tabou faisant comprendre à sa belle-sœur que sa présence était indésirée: propos humiliants, menaces verbales indirectes, évocation indirecte de sa sorcellerie, etc., la peur étant mauvaise conseillère, Tabou développe diverses formes de violence psychique à l'encontre de Kato, en traitant, entre autres, son fils présumé sorcier de chien galleux. Kato à son tour indiquait par une attitude hautaine et de mépris ostentatoire qu'elle était chez son frère et que personne ne l'en ferait partir. Elle s'immisça dans les conflits du couple, prenant le parti de son frère et insultant Tabou pendant leurs altercations. Pour couronner le tout, véritable torture psychologique, Kato s'allia à la rivale de Tabou, la maîtresse de son frère, au vu et au su de Tabou. Dans cette séquence, les éléments d'un échange généralisé de violences apparaissent clairement.

Une dizaine d'années plus tard, la situation s'inversa. C'est Tabou qui se retrouva hébergée par Kato. En effet, l'enfant maladif, présumé sorcier, mourut en 1994. Kato se remaria en 1996. Kola perdit son emploi en 1997 et Tabou perdit le sien en 1998. Sans revenus, incapables de payer leur loyer, ils se réfugièrent chez Kato en 1998. Kola partit à la recherche de diamants en Angola en 1999. Lorsque je fis mes enquêtes dans cette famille en juin 2001, Tabou attendait toujours un retour improbable de son mari. Hébergée par sa belle-sœur Kato, sans revenus sûrs, celle-ci à son tour, lui en faisait voir « de toutes les couleurs ».

Outre les formes de résistance directe ou indirecte contre la violence, ceux qui se trouvent au bas de la hiérarchie subissent aujourd'hui une violence qu'ils pourront faire subir demain à d'autres. Sur le plan synchronique, les victimes des violences familiales, tendent de plus en plus à répondre à la violence par la violence, comme dans le cas de Kato et Tabou. Sur le plan diachronique, les cadets et les faibles d'hier, étant les aînés et les puissants de demain, ce changement de position contribue à la reproduction de la violence, à son caractère cyclique, et à la perpétuation des rapports de violence. ${ }^{18}$ Toute position structurellement dominante dans la famille étant relative et réversible, la violence familiale ne peut qu'être réciproque. (Janin et Marie 2003) Ceux qui subissent la domination tentent parfois d'inverser les rapports de pouvoir, mais plus généralement ils attendent d'être à leur tour en position de pouvoir pour dominer les autres. Il ne s'agit pas seulement d'une reproduction mimétique des actions de violence que l'on aurait subies ou observées dans l'enfance, mais de la reproduction et de la perpétuation des rapports de domination, fortement hiérarchisés dans la structure familiale, rapports à travers lesquels s'exprime et se perpétue la violence familiale.

Violences réciproques, violences cycliques, violences infinies 
27 Au-delà de la violence quotidienne dans les rapports familiaux, il y aurait sans doute beaucoup à dire sur la violence à Kinshasa en général, dans le domaine politique ou criminel. Mais ce n'était pas mon propos. Je voudrais simplement indiquer à ce sujet, qu'après une longue période de dictature dans le pays, l'on observe à Kinshasa depuis les débuts des années 90' ouvrant une période de transition à la "démocratie ", caractérisée par des luttes pour le pouvoir politique, des manifestations de troubles et de violences publiques, une montée de la violence sociale et politique: pillages, jacqueries, émeutes, violences étudiantes, etc. (de Villers et Omasombo 2004, Devisch 1998, Ndaywel 1998). Ville mégalopole, Kinshasa connaît par ailleurs une montée spectaculaire des formes de violence urbaine telle que la criminalité, la délinquance juvénile, le banditisme armé, etc. qui, selon la thèse de Pérouse de Montclos (2002), accompagnent toujours la croissance urbaine et en fait partie intrinsèquement. D'autres violences de rue, tensions et conflits, sont également liées au grand essor de la musique populaire à Kinshasa (Tsambu 2004). En somme, l'on peut dire avec de Villers et Omasombo qu'il se développe à Kinshasa une "violence nue (haineuse, cruelle, destructrice, jouisseuse) (...) violence contre les biens, contre les symboles, contre les personnes; elle s'en prend aux vivants et aux morts ». (2004:226) La violence urbaine que l'on observe à Kinshasa est donc multiforme.

Ces différentes formes de violence urbaine ne sont pas sans liens comme on pourrait le croire, avec les violences dans les rapports familiaux quotidiens analysées ici. La montée actuelle de la violence urbaine à Kinshasa, particulièrement le banditisme armé et la délinquance juvénile est, entre autres, liée à l'augmentation extraordinaire du nombre des enfants de la rue. Ces derniers s'y retrouvent pour la plupart, parce qu'ils sont désignés par les familles comme enfants sorciers (De Boeck 2000). Violentés, chassés, par les familles, ils se retrouvent dans la rue et grossissent de ce fait, les rangs des délinquants juvéniles. Ils sont alors « initiés » à la violence de la rue, et deviennent de véritables acteurs de la violence urbaine..$^{19} \mathrm{~L}$ 'on peut ainsi voir l'une des articulations de la violence de la sphère familiale à la violence de la sphère publique, et comment l'une peut contribuer à renforcer l'autre. Les violences dans les rapports sociaux au quotidien sont aussi l'expression des représentations sociales d'une société où l'infortune, la maladie, ou la mort sont attribuées à l'action des personnes malfaisantes comme les sorciers; d'une société où selon Alain Marie, le sort et l'identité de chacun sont rivés pour le meilleur et pour le pire, aux bons ou mauvais vouloirs, aux dispositions fluctuantes de son entourage social et surréel (1997: 323). Aussi déclenchent-elles de plus en plus en ville, une « chasse » aux maléfices et aux sorciers, dans une sorte de paranoïa généralisée. Dans un contexte urbain en crise où chacun essaie de "sauver sa peau » aux dépens de l'autre, la dureté dans les rapports sociaux quotidiens a tendance à s'exacerber, et amène de plus en plus à questionner le lien familial et son devenir, avec la persistance et l'aggravation de la crise. Malgré les apparences de convivialité, que l'on peut observer dans les liens familiaux en milieu urbain, ils sont en même temps et de plus en plus ambivalents, c'est-à-dire fondés à la fois sur une grande affectivité, une conscience du besoin de solidarité, et sur une méfiance réciproque et une suspicion mutuelle. Ils ne peuvent par conséquent qu'être chargés de potentiels de violences silencieuses ou explicites, où jalousie, concurrence, vengeance et exclusions peuvent s'entremêler.

29 Il me semble finalement important de souligner qu'il ne peut y avoir de responsabilité établie une fois pour toutes dans la répartition et la diffusion des violences 
quotidiennes dans les rapports familiaux (Janin 2003), tellement enracinées dans les pratiques quotidiennes qu'elles tendent à être banalisées, minimisées. ${ }^{20}$ Elles trouvent leur enracinement dans la longue durée historique et se déploient aisément grâce à l'autoritarisme de l'ordre communautaire organisant la vie familiale. Aussi, la «tradition» qu'on évoque parfois pour justifier certaines pratiques ou attitudes de violence, ne constitue souvent qu'un outil aux mains des puissants et des aînés pour perpétuer un rapport de force en leur faveur. Les deux cas évoqués ici montrent bien que la position dominée dans la hiérarchie familiale n'explique pas à elle seule la situation de victime. Il y a aussi un facteur déterminant à la vulnérabilité ou à la réversibilité des situations de violence tel que l'a illustré l'inversion finale de la situation entre Kato et Tabou: c'est la précarité économique. De plus en plus, le dominant est dominant à cause de sa relative sécurité économique, c'est ainsi que le dominé statutaire peut se défendre contre la violence d'un dominant statutaire, mais économiquement affaibli, comme Kato avec son emploi contre son mari. Le dominant statutaire/dominé économique n'a plus que la violence de sa frustration pour faire prévaloir son « autorité ».

La résistance à certaines formes de violence familiale semble possible lorsqu'on dispose de moyens économiques, qui peuvent permettre par exemple de s'installer loin des beaux-parents, de se «libérer » de la torture «traditionnelle» des belles-sœurs aux funérailles, de se « libérer » d'un mauvais sort (tel que le mari de Chantal qui paye une nouvelle dot), au couple de rester ensemble, etc. Dans les deux cas étudiés, deux types de facteurs interagissent étroitement: le statut social (position hiérarchique dans l'ordre d'aînesse ou de genre) et le statut économique; et il semble bien que c'est la précarité économique qui expose le plus au risque de la violence statutaire. Dans le jeu compliqué de l'inversion des rôles de domination/soumission statutaire comme dans celui d'empêcher la vengeance sorcière, la prospérité/précarité économique semble jouer un rôle régulateur.

31 En conclusion je voudrais dire combien la violence sociale et relationnelle actuelle est signe et symptôme d'une société en crise très profonde où la violence était déjà favorisée historiquement dans les attributs d'autorité des rapports sociaux dominants. L'anomie que l'on observe aujourd'hui tient en partie au fait que les mécanismes légitimes de régulation de contrôle (droit, coutume, institution judiciaire et policière) sont dans l'incapacité de jouer leur rôle à cause, entre autres, de la généralisation de la menace de sanction sorcière qui empêche tout recours citoyen à ces formes de juridiction et de coercition légales.

\section{BIBLIOGRAPHIE}

Abega Séverin Cécile, 2003, «La violence endémique en Afrique. Editorial », in Bulletin de l'APAD «La violence endémique en Afrique », $\mathrm{n}^{\circ} 25, \mathrm{p} .5-7$.

Dauphin Cécile, Farge Arlette (éds), 1997, De la violence et des femmes. Paris, Albin Michel. 
De Boeck Filip, 2000, « Le deuxième monde et les enfants sorciers en RDC », in Politique Africaine «Enfants, jeunes et politiques"», n 80, p.32-57.

De Villers Gauthier, Omasombo, Jean, 2004, « Quand le peuple kinois envahit les rues...», in " Ordre et désordre à Kinshasa. Réponses populaires à la faillite de l'Etat », Cahiers africains, $\mathrm{n}^{\circ}$ 61-62, p.213-233.

Devisch René, 1998, « La violence à Kinshasa ou l'institution en négatif », in Cahiers d'études africaines, « Disciplines et déchirures. Les formes de la violence », 150-152, XXXVIII (2-4), 475-495.

Dissisa Vincent, 2003, « Violence et funérailles au Congo-Brazzaville », in Bulletin de l'APAD «Violence endémique en Afrique », $\mathrm{n}^{\circ} 25, \mathrm{p} .89-97$.

Geschiere Peter, 1995, Sorcellerie et politique en Afrique. La viande des autres. Paris, Karthala. Gillioz Lucienne, De Puy Jacqueline, Ducret Véronique, 1997, Domination et violence envers la femme dans le couple. Lausanne, Payot.

Girard René, 1993, La violence et le sacré. Paris, Grasset.

Girard René, 2003, Celui par qui le scandale arrive. Paris, Desclée de Brouwer.

Janin Pierre, 2003, «Vivre ensemble ou la douleur d'être en grande famille », in Politique Africaine, «Violences ordinaires », $n^{\circ}$ 91, p. 33-50.

Janin Pierre, Marie Alain, 2003, « Violences ordinaires, violences enracinées, violences matricielles », in Politique Africaine, « violences ordinaires », n 91, p.5-12.

Jaspard Maryse, 2005, Les violences contre les femmes. Paris, La Découverte.

Lenclud Gérard, 1994, « Qu'est-ce que la tradition », in Transcrire les mythologies. Paris, Albin Michel.

Marie Alain, 2003, « La violence faîte à l'individu », in Politique Africaine, « Violences ordinaires », $n^{\circ}$ 91, p. 13-32.

Marie Alain, 1997, « Avatars de la dette communautaire. Crises des solidarités, sorcellerie et procès d'individualisation ", in MARIE, Alain (éd), L'Afrique des individus. Paris, Karthala, p. 249-328.

Ndaywel è Nziem Isidore, 1998, « Du Congo de la rébellion au Zaïre des pillages », in Cahiers d'études africaines, « Disciplines et déchirures. Les formes de la violence », 150-152, XXXVIII (2-4), 455-474.

Ngindu Alphonse, 1969, « Propos et problèmes concernant le culte des morts chez les Baluba du Kasaï », in Cahiers des religions africaines, vol 3, n 5, p.25-35.

Perouse de monclot Marc Antoine, 2002, Villes et violences en Afrique noire. Paris, IRD/Karthala.

Tsamby bulu Léon, 2004, « Musique et violence à Kinshasa ", in « Ordre et désordre à Kinshasa. Réponses populaires à la faillite de l'Etat », Cahiers africains, n 61-62, p.194-212.

\section{NOTES}

1. Il existe un certain nombre d'études qui ont été menées, particulièrement dans les pays occidentaux, à propos des violences contre les femmes. Dans un ouvrage récent, Maryse Jaspard (2005) fait une synthèse des travaux consacrés aux violences contre les femmes en général, et en particulier aux femmes en situation de subordination vis-à-vis 
de leur conjoint ou de leur famille. L'on peut y trouver les principales références sur le sujet et une présentation des principaux résultats des récentes enquêtes.

2. J'entends par cycle "la fluctuation d'un phénomène qui se compose de phases d'expansion suivies de phase de récession, de contraction et de reprise, engendré par un effet de rétroaction négative". (Boudon Raymond et alii 1989, Dictionnaire de la sociologie. Paris, Larousse : 54.

3. Les récits de vie proposés dans cette contribution, ont été collectés à Kinshasa en 2000 et 2001, dans le cadre d'une recherche personnelle sur "les églises néo-pentecôtistes et la question de la solidarité familiale". Ils ont été complétés dans le cadre d'une A.C.I. en cours (2003-2006) soutenue par le Ministère français de la recherche, CNRS/IEA.

4. A Kinshasa, il est courant que les parents des jumeaux soient appelés respectivement, Papa (ou Tata) et Mama Mapasa. Le mot Mapasa (singulier Lipasa) signifie jumeaux en lingala. C'est un pseudonyme choisi par respect de la clause de confidentialité, clause à laquelle obéissent tous les autres noms de personnes proposés dans cette contribution. Cf. Bertaux, Dominique, 2003, Les récits de vie. Paris, Nathan. 5. Les pratiques du bain " purificateur » et autres cérémonies du rituel funéraire destinées à libérer la veuve de son lien avec le défunt pour réintégrer la société après le deuil, étaient très variables dans les contextes traditionnels, - des différentes ethnies au Congo. Pour aider le lecteur à avoir une idée de comment se passaient les deuils en milieu traditionnel et de mieux comprendre le prolongement de certaines de ces pratiques en milieu urbain, voici ce qu'Alphonse Ngindu rapporte à propos des Baluba du Kasaï par exemple, groupe ethnique auquel appartient la famille de Papa Mapasa : "Les proches parents du défunt se rasent complètement la tête en signe de tristesse. Durant tout le deuil, les frères du défunt et leurs sympathisants couchent à la belle étoile. Les femmes quant à elles couchent à l'intérieur de la maison mortuaire à même le sol ou sur des nattes. Toutefois, c'est la veuve qui porte vraiment le deuil. C'est l'occasion pour elle ou jamais de montrer l'amour et l'attachement qu'elle portait à son mari disparu. Elle doit pleurer son mari selon l'expression consacrée : tête rasée, corps presque entièrement nu et couvert de cendres. Elle pleure jour et nuit, parle peu ou point, refuse les soins corporels : toilette, manger, boire, tout cela est réduit au strict nécessaire (...) le jour anniversaire, une semaine, ou plusieurs mois après, suivant les situations, on procède à la levée du deuil (...) La levée du deuil est toujours un grand événement dans un village. C'est la reprise de la vie. On tire des coups de fusils, on chante et on danse toute la nuit. (...) Le rituel funéraire des Baluba (...) semble avoir à leurs yeux un double caractère. D'une part, les proches parents ont conscience d'être contaminés, profondément affectés dans leur existence par la mort d'un des leurs. Ils doivent être purifiés (...) D'autre part, le mort s'accroche aux vivants. L'homme décédé (...) continue à roder autour du village. Il tente de retrouver sa maison et sa couche. Il apparaît en rêve. Il effraie les passants sur les sentiers (...) Les rites de purification dont nous avons parlé visent précisément une séparation des vivants avec le mort, en particulier de la veuve avec son époux ». (1969: 90-91, 93)

6. Pendant la colonisation, la création des villes et le brassage interethnique qui en a résulté avec le temps, ont occasionné un métissage des rituels funéraires qui se sont prolongés et maintenus dans le contexte urbain. A Kinshasa, ils tendent depuis quelques années à s'estomper dans certaines familles. Mais dans d'autres, sans disparaître totalement, ils tendent à être simplifiés et réduits au strict minimum. 
L'hyper religiosité qui règne dans la ville depuis quelques années, mais surtout la crise socio-économique ne sont sans doute pas, étrangères à ce recul des rites funéraires.

7. La violence pendant les funérailles à Kinshasa ne touche pas seulement la maltraitance des veuves. Les funérailles sont depuis quelques années, des lieux où s'expriment plusieurs formes de violence, notamment sous forme d'agressions physiques ou verbales envers des personnes soupçonnées de sorcellerie, ou désignées comme ayant "mangé" le défunt. L'on observe également lors des funérailles à Kinshasa, comme le décrit Vincent Dississa (2003) pour le contexte très proche du Congo/Brazzaville, une forme de violence yankiste, de l'expression locale " yanké », désignant des jeunes délinquants, voyous, brigands, ou malfaiteurs. Durant les funérailles, ces “yankés” ont tendance à envahir l'espace funéraire, à transgresser certains interdits, tels que fumer publiquement du chanvre, proférer des propos grossiers, scander des chants vulgaires évoquant les parties génitales du corps, insulter et menacer en permanence les adultes, surtout les vieillards, détourner le parcours du cortège funèbre, rançonner les passants, etc.

8. A propos de la perpétuation des rites traditionnels, Gérard Lenclud indique que le rite répète, tout en accommodant son accomplissement de biens des libertés; il n'est donc pas du domaine de la réitération exacte. "Les historiens des sociétés rurales ont établi que les coutumes n'ont pas fixé d'anciennes traditions, mais mis en forme des institutions d'apparition tardive, parées par la grâce (tradionnante) de l'écrit d'un vêtement archaïque. Bref, les traditions, autant que les traductions, sont des belles infidèles ». (1994 : 30) Une tradition s'invente ou se recrée, et elle donc une réponse trouvée dans le passé à une question formulée dans le présent, et elle exhibe un morceau de passé taillé aux mesures du présent dans un atelier contemporain. (1994 : 33-34)

9. Cette cour familiale regorgeait de monde. Papa Mapasa était issu d'une famille de douze enfants dont il était huitième. Ses frères et sœurs aînés y habitaient avec leurs conjoints et leurs enfants, sans compter des cousins, cousines, oncles, tantes, et autres parents classificatoires.

10. Par exemple, lorsque Mama Mapasa lui présentait à manger, soit elle disait que sa belle fille ne savait pas faire la cuisine, soit elle refusait dédaigneusement en disant que la portion était insuffisante. Lorsque Mama Mapasa ne lui présentait pas à manger, elle disait à tous, que son fils avait épousé une vipère qui laissait sa belle-mère mourir de faim. Mais la belle-mère était plutôt conciliante avec ses autres belles-filles dont elle avait approuvé le choix, ou qu'elle avait elle-même choisi.

11. Comme le notent Dauphin et Farge (1997), en raison de leur rôle d'éducatrice au sein de la famille, les femmes inculquent, perpétuent, reproduisent, les rapports sociaux de pouvoir, entre aînés et cadets, dominants et faibles, ayant elles-mêmes assimilées, les contraintes liées à leur statut inégalitaires par exemple.

12. La sorcellerie est parfois désignée dans l'imaginaire populaire à Kinshasa comme quelque chose qui réside dans le ventre. Dans le langage courant, l'on entend dire de quelqu'un qu'il aurait “quelque chose dans le ventre" pour désigner qu'il est un sorcier potentiel. Ce mode de désignation de la sorcellerie semble assez commun en Afrique centrale. Dans ses travaux sur la sorcellerie au Cameroun (chez les Maka), Peter Geschiere (1995) rapporte des expressions semblables.

13. Il convient d'indiquer ici pour éviter toute confusion, que les choses ne se passent pas toujours ainsi dans toutes les familles, puisque les veuves et orphelins ne sont pas toujours dépouillés de l'héritage. Il faudrait indiquer aussi qu'il existe effectivement 
des veuves et orphelins qui ne se laissent pas faire, surtout lorsque les orphelins sont déjà adultes et responsables dans la société. Il arrive parfois que les affaires soient déférées devant les tribunaux lorsque les tentatives de règlement en famille n'aboutissent pas.

Il est cependant difficile de s'avancer dans le cadre de ce travail sur le niveau de généralisation de ce phénomène de dépouillement de l'héritage dans une ville comme Kinshasa. Mais il reste qu'il est assez courant d'observer des veuves et orphelins se disputer l'héritage avec la famille d'origine du défunt, ou se faire complètement dépouiller de l'héritage. D'après certains de mes informateurs, le phénomène s'observerait depuis environ trente ou quarante ans, d'abord dans les familles appartenant à des ethnies à système matrilinéaire. Les difficultés financières dans les quelles vivent les familles dans la ville de Kinshasa depuis quelques années à cause de la grave crise socioéconomique contribue certainement à son amplification, même chez ceux qui appartiendraient à des ethnies à système patrilinéaire.

14. Le « blocage » est un terme généralement utilisé dans les milieux des églises indépendantes à Kinshasa, pour désigner les freins à la réussite matérielle et financière d'une personne. Ces freins seraient parfois dus à l'action des personnes malfaisantes ou jalouses, telles que les sorciers, ou recourant aux services des personnes ayant des pouvoirs surnaturels, tels que les féticheurs, pour entraver la réussite des autres. 15. Les résultats des principales enquêtes sur les violences conjugales présentés en synthèse par Maryse Jaspard (2005) sont éclairants. Ils montrent que, même dans les sociétés occidentales, les violences conjugales ne sont pas négligeables. Ces violences conjugales en occident, dans un contexte social prohibant la violence physique, ne se limitent pas seulement au fait de battre les femmes; les violences (pressions) psychologiques y sont prépondérantes. Dans une autre étude, consacrée au cas spécifique de la suisse par exemple (Gillioz, DePuy \& Ducret, 1997), les auteurs rendent comptent de l'ampleur des violences conjugales envers les femmes dans ce pays, tout en mettant en évidence les facteurs sociaux et familiaux liés à cette violence.

16. En ce qui concerne par exemple les violences conjugales et leurs limites " acceptables ", une femme me confiait lors d'un entretien en mai 2004 que pour elle, il était normal, et même bon, qu'un homme batte de temps en temps sa femme, sans " dépasser certaines limites » ou " sans lui faire trop mal », car c'est une façon de lui prouver qu'il l'aime et qu'il tient à elle. Elle soutenait que lorsqu'un homme ne bat plus sa femme, il est probable qu'elle ne l'intéresse plus ou qu'il ne l'aime plus. En appui à son point de vue, elle m'a fredonné les paroles d'une chanson populaire, qui reprend un adage selon lequel « Les coups dans un mariage sont des preuves d'amour ».

17. Il est communément admis, dans les conceptions sociales à Kinshasa, que la sorcellerie est transmissible, « contagieuse », surtout chez des jeunes enfants. Elle est souvent décrite comme une "maladie" (sous-entendue de l'âme) que l'on peut contracter, soit par hérédité, soit par contagion, que l'on peut à son tour transmettre, et dont on peut guérir si l'on reçoit un traitement approprié, et si l'on a la volonté de s'en débarrasser. D'où la "théorie" de la sorcellerie inconsciente évoquée précédemment, selon laquelle, une personne pourrait contracter la sorcellerie sans le savoir, et donc être sorcière à son insu.

18. S'interrogeant sur le caractère endémique de la violence et sur la perpétuation de la violence, Séverin Abega (2003) se demande lui aussi à juste titre, comment celle-ci se maintient, se répète, se répand, et indique qu'il est important pour arriver à le 
comprendre, d'arriver à décrire correctement à partir des travaux de terrain les mécanismes de sa perpétuation.

19. Voir dans ce numéro la contribution sur l'initiation à la violence des enfants de la rue à Lubumbashi.

20. Peut être faudra t-il évoquer ici la thèse de René Girard $(1993,2003)$ sur le désir mimétique de l'homme pour expliquer la violence. Selon lui, le sacrifice rituel de la victime émissaire, qui est au centre de la religion, « fonde » également la sociabilité, et ce mécanisme fondateur est toujours à l'œuvre quel que soit par ailleurs, le niveau de complexité des phénomènes sociaux évoqués. Selon lui, la base sociale est donc religieuse, et le sacré « est tout ce qui maitrise l'homme d'autant plus sûrement que l'homme ne se croit plus capable de le maîtriser » (1993:52), mais le désir humain, qui inclut une tendance essentielle à imiter l'autre, entretient une violence impure, sans cesse recommencée. Aussi pense $t$-il que seul le lien religieux peut éloigner le recours fondateur à la victime expiatoire, et peut-être même désamorcer la violence de notre époque.

\section{RÉSUMÉS}

Cette contribution se penche sur l'aspect réciproque et cyclique des violences dans les rapports familiaux en milieu urbain africain, en investiguant particulièrement le rapport épouse/belle famille. A travers ce rapport, c'est d'abord le lien familial et ses violences associées qu'elle voudrait interroger, mais aussi la reproduction et la perpétuation des rapports de violence dans le contexte familial. En analysant les violences quotidiennes, ordinaires, des rapports familiaux dans une perspective diachronique, elle tente de dépasser un discours qui pourrait ériger les uns en agresseurs exclusifs et les autres en victimes exclusives, et de montrer par contre, que dans la longue durée d'un cycle d'échanges de violences chacun à son tour peut être victime et oppresseur.

Title : Social Violence \& mutual exclusions in Urban Africa. The Cyclic Exchange of Violence among kins. This paper tries to highlight the reciprocal and cyclic dimension of ordinary violence occurring in urban African kinship relationships. Through the description of two case studies relating life histories, it investigates a peculiar type of family tie : the wife and mother in law violent relationship. But through this relationship, it is first the kinship link and its associated violence that are investigated and second, the way by which violent relationships reproduce and perpetuate themselves in the family context. By analyzing those usual and ordinary violence of kin relationships from a diachronic point of view, we attempt to overtake the oversimple view that would consider some kin as exclusive aggressor while the other would stay as exclusive victim. On the contrary, the cases observed shows that in the long run of a cycle of violence exchanges anyone may be in turn victim or oppressor. 